\title{
On the measurement bias of low- $\ell$ solar $p$-mode excitation parameters: The impact of a ground-based window function
}

\author{
W. J. Chaplin ${ }^{1}$, Y. Elsworth ${ }^{1}$, G. R. Isaak ${ }^{1}$, B. A. Miller ${ }^{1}$, R. New ${ }^{2}$, B. Pintér ${ }^{2}$, and S. Thiery ${ }^{1,3}$ \\ 1 School of Physics and Astronomy, University of Birmingham, Edgbaston, Birmingham B15 2TT, UK \\ 2 School of Science \& Mathematics, Sheffield Hallam University, Sheffield S1 1WB, UK \\ 3 Institut d'Astrophysique Spatiale, Université Paris XI, 91405 Orsay, France
}

Received 20 August 2002 / Accepted 6 November 2002

\begin{abstract}
We present a study of the impact of a ground-based, multi-station window function on estimates of the power and damping of low- $\ell$ solar $p$ modes extracted from fits to resonant structure in the frequency domain. The window functions come from six-site observations made by the Birmingham Solar-Oscillations Network (BiSON) over the 10-yr period beginning 1991 January. Two strategies were adopted. In the first, we used an 800-d time series of continuous observations made by the GOLF instrument on board the ESA/NASA SOHO satellite. These data were modulated by a variety of BiSON window functions, with fractional duty cycles ranging from $\sim 0.4$ to $\sim 0.8$, and the resulting series analyzed. In the second we generated artificial 10 -yr time series and studied the effect on these of the complete BiSON window.
\end{abstract}

Key words. methods: data analysis - Sun: oscillations

\section{Introduction}

Solar $p$ modes are believed to be excited stochastically by turbulence in the outer layers of the convection zone (for recent theory see, e.g., Houdek et al. 1999; Houdek 2001; Stein \& Nordlund 2001; Samadi et al. 2001). Insight into the excitation and damping processes can be acquired through the study of observations of the resonant mode profiles. In particular: the frequency-domain linewidth provides a direct measure of the damping of the mode; the maximum height of the profile a measure of the balance between the excitation and damping; and the peak asymmetry information regarding the localization and nature of the excitation source.

Here, we present a study of the impact of a ground-based, multi-station window function on estimates of the width and height parameters of globally coherent, low-angular degree $($ low $-\ell$ ) modes. The most prominent of these have radial overtone numbers of the order of $n \sim 20$. Since departures from the Lorentzian form expected of a damped, stochastically driven oscillator are rather small, the asymmetry (typically of the order of one to two per cent at low $\ell$ ) has a rather modest influence on the magnitude of the extracted width, $\Delta v_{n \ell}$ and height, $H_{n \ell}$. We leave a discussion of the problems associated with fitting this parameter to another paper.

Send offprint requests to: W. J. Chaplin, e-mail: wjc@bison.ph.bham.ac.uk
The importance of being able to obtain accurate estimates of the excitation parameters is vital, not only for informing our understanding of the interaction of the acoustic waves with their environment in the outer layers of the Sun, but also for making predictions of the oscillation spectra of other stars (e.g., Houdek et al. 1999). Here, the two fundamental, and independent, quantities that any full description must model are the damping constant, $\eta$, and the acoustic noise generation rate, $P_{Q}=\mathrm{d} E / \mathrm{d} t$ (sometimes also called the energy supply rate). A radial mode with associated inertia $I_{n \ell}$ is then predicted to have an observed integrated velocity power of:

$V_{n \ell}^{2}=\frac{K_{\theta, \phi} P_{Q}}{2 \eta_{n \ell} I_{n \ell}}$

where $K_{\theta, \phi}$ is some suitable correction to allow for the spatial filter of real observations. When a Lorentzian resonance is assumed - a not unreasonable assumption given the small peak asymmetry - the height and width parameters imply that the power can also be expressed observationally as:

$V_{n \ell}^{2}=\frac{\pi}{2} \Delta v_{n \ell} H_{n \ell}$.

(This assumes the mode to be resolved across several bins: see Appendix A.) Since the damping constant can be measured directly through the linewidth, i.e.,

$\eta_{n \ell}=\pi \Delta v_{n \ell}$, 
Eqs. (1) and (2) imply that an observed estimate of the acoustic noise generation rate can be made from:

$P_{\mathrm{Q}}=\mathrm{d} E / \mathrm{d} t=\left(\frac{\pi^{2} I_{n \ell}}{K_{\theta, \phi}}\right) \Delta v_{n \ell}^{2} H_{n \ell}=\left(\frac{2 \pi I_{n \ell} V_{n \ell}^{2}}{K_{\theta, \phi}}\right) \Delta v_{n \ell}$

This is partially model dependent through predictions of the $I_{n \ell}$ and $K_{\theta, \phi}$. Of most importance here, Eq. (4) implies that any observational estimate of $P_{\mathrm{Q}}$ demands separate estimates of $\Delta v_{n \ell}$ and $H_{n \ell}$, or at the very least an independent estimate of $\Delta v_{n \ell}$ if an integrated measure of the velocity power can be made.

The usual method by which estimates of the linewidth and peak height are extracted is by fitting the resonant structure to a suitable model in the frequency domain. Careful correction must in principle be made for the deleterious effects of a ground-based window function (Chaplin et al. 2000; Komm et al. 2000). Here, we perform an in-depth study of the impact of a variety of ground-based windows with fractional fills ranging from $\sim 0.4$ to $\sim 0.8$. These are constructed from coherent combinations of observations made by the six-station Birmingham Solar-Oscillations Network (BiSON). We quantify the effect of these on: (i) 800-d of continuous low- $\ell$ observations made by the GOLF instrument on board the ESA/NASA SOHO satellite; and (ii) artificially generated datasets of length up to $10 \mathrm{yr}$. The former allows us to test directly the analysis with real data for a time series length that is sufficient to study subtle effects in the spectrum (e.g., peak asymmetry, $\ell=2$ multiplet frequency asymmetry), at the same time giving some temporal resolution over the 11-yr solar activity cycle; while the latter approach allows us to check the analysis on timescales comparable to the period of the cycle.

Our aim is primarily one of assessing the extent of any bias present in fitted estimates of $\Delta v_{n \ell}$ and $H_{n \ell}$. For analyses involving artificial data the unbiased parameter values are those that are used to construct the data. Analyses made with the GOLF data allow a direct measure of parameter bias with respect to those estimates given from the continuous set. These are of course not necessarily the same as the actual solar values, although the simulations do allow us to address the extent to which they might differ. Differences in observational technique between BiSON and GOLF should also be borne in mind.

Each BiSON spectrometer determines the disc-averaged Doppler shift of the potassium Fraunhofer line at $770 \mathrm{~nm}$ (e.g., Chaplin et al. 1996). This is achieved by making nearsimultaneous measures of the intensity in two passbands at fixed (with respect to a laboratory reference) wavelength that are located in the blue and red wings of the Fraunhofer line respectively. The normalized difference of these provides a nearlinear measure of the relative line-of-sight Doppler shift of the solar surface. In contrast, the GOLF data used here were collected while it measured the intensity in the blue wing only of the sodium D1/D2 Fraunhofer doublet. As such there may be subtle differences in, for example, the amplitudes, backgrounds and small peak asymmetries measured by BiSON and GOLF owing to: the different heights sampled in the solar atmosphere, and the differing sensitivity to pure intensity fluctuations that may modify the pure Doppler signal (e.g., Pallé et al. 1999; Jiménez et al. 1999).

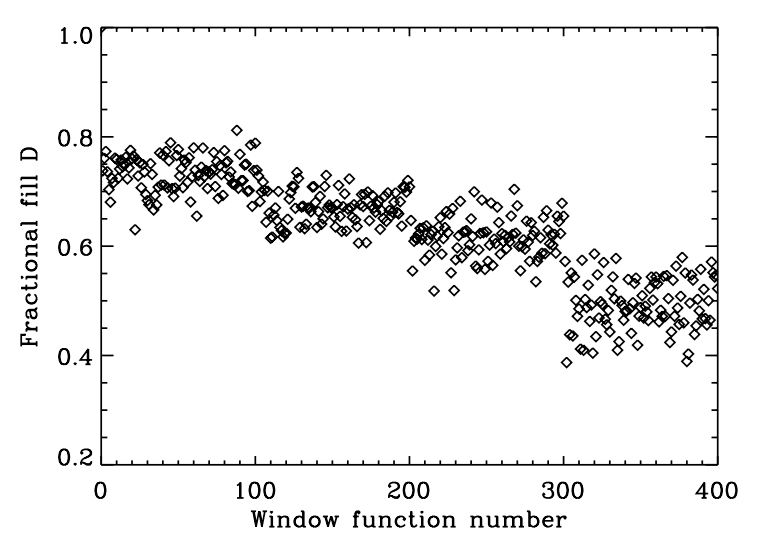

Fig. 1. Duty cycles of the 800-d BiSON window functions used to modulate the continuous GOLF time series. Each was made from the random combination of eight 100-d parts taken from four 10-yr BiSON time series. The 10-yr series were made from a coherent combination of data from different numbers of stations (varying from three to six sites). The discontinuities in the plot reflect the varying number of sites used.

\section{Data and fitting analysis}

\subsection{Modulation of GOLF data by BiSON window functions}

For our first strategy we made use of 800 -d of data collected between 1996 April and 1998 June by the GOLF instrument on board the ESA/NASA SOHO satellite. This period covers an epoch of low activity near the transition between activity cycles 22 and 23. The GOLF time series has excellent continuity (better than 99 per cent) and can be regarded therefore as having an effective 100 -per-cent duty cycle $(D=1.00)$. These data were first re-binned to a 40-s sampling (to match that of the BiSON data). The re-sampled set was then modulated by a whole series of observational BiSON window functions, with each power spectrum being fitted to yield estimates of the width and height parameters under conditions of varying fill (in the usual manner described by, for example, Chaplin et al. 1999, 2002).

To make the various 800 -d windows we used not only the full six-station BiSON time series, but also sets made from coherent combinations of data collected by three-, four- and five-station combinations only. This gave us access to lower duty cycles not available in the six-station configuration. Each multi-site window function was divided into thirty six 100-d pieces; random 8-piece combinations of these were then made giving in all four hundred 800-d window functions with fills varying from $D \sim 0.4$ to $\sim 0.8$. The duty cycles of these are plotted in Fig. 1. The discontinuities arise from the different numbers of stations used to make sets.

\subsection{Artificial time series}

We have used the Laplace transform solution of the equation of a forced, damped harmonic oscillator to generate artificial $p$-mode-like data in the time domain (Chaplin et al. 1997) that could be modulated by the real BiSON window functions. 
The simulated data were made on a 40 -s cadence in order to match that of the BiSON observations. All $m$ components to which the full-disc observations are sensitive were represented explicitly in each complete time series set as an oscillation signal generated by a single "run" of the model. Each component was re-excited continually by small "kicks" at each 40-s sample. Furthermore, we adhered to a stochastic description that assumed each mode was excited independently. This was realized in practice by randomly phasing, in time, all generated signals. Each set was made with a full cohort of simulated low- $\ell$ modes, covering the ranges $0 \leq \ell \leq 5$ and $1000 \leq v \leq 5200 \mu \mathrm{Hz}$. A "pink" background noise component was added also in the time domain, the size of which increased at lower frequencies in order to give realistic signal-to-background ratios. We constructed artificial sets of length $3456 \mathrm{~d}(\sim 10 \mathrm{yr})$ and $800 \mathrm{~d}$ (the latter to allow a direct comparison with the GOLF time series).

We used a database of mode frequency and linewidth estimates, as obtained from analyses of BiSON spectra, in order to fix the characteristics of each oscillator component (i.e., its resonant frequency and characteristic damping constant and power). Visibility levels for the barely-detectable $\ell=4$ and 5 modes were fixed at the levels calculated by Christensen-Dalsgaard (1989). At the extreme ends of the modeled spectra - where we do not have reliable, fitted estimates for the parameters - we made appropriate, low-order extrapolations of the parameter values from the well-studied part of the spectrum.

In our base level simulations, all multiplets were constructed with a symmetric rotationally induced splitting pattern with a synodic spacing between adjacent $m$ components of $400 \mathrm{nHz}$. This value was chosen in order to match values of the splittings extracted from fitting real data at low $\ell$.

The model gives rise to a limit spectrum that takes a Lorentzian form for each resonant mode in the frequency domain. As such, it provides no representation of the small peak asymmetries that have now been uncovered at low $\ell$ (e.g., Toutain et al. 1998; Chaplin et al. 1999; Chaplin \& Appourchaux 1999; Thiery et al. 2000; Bertello et al. 2000). While we accept that this is a shortcoming of the simulations, the small size of the asymmetries is such that they should have only a modest impact on the extracted width and height parameters. We therefore expect the conclusions drawn from the artificial data to be robust.

We nevertheless included a free parameter to allow for any peak asymmetry when fitting the artificial spectra. This ensured we varied the same number of free parameters in fits to both the real and simulated data (i.e., the asymmetry is fitted when the GOLF and real BiSON sets are analyzed). This is a subtle but important point - the distribution of fitted estimates of a parameter can be affected by the number of other parameters that we seek to estimate at convergence. If one of the varied parameters is poorly constrained the number of iterations required to reach convergence may be affected adversely, giving (unwanted) increased scatter in those parameters that are more strongly correlated with it. Here, we aim to have our simulations reflect these difficulties. We note in passing that fits to the artificial data extracted peak asymmetries consistent with a zero value.



Fig. 2. The effects of a 75 -per-cent-fill window function in the frequency domain. Here, 1000 independent realizations of the same, artificial $l=2, n=18, l=0 n=19$ mode pair - with each component possessing an underlying Lorentzian limit profile - were generated in the time domain. The solid line shows the mean power spectrum obtained by adding the independent frequency spectra, with each time string having first been modulated (in the time domain) by an 8-month BiSON window function; the dashed line illustrates the mean spectrum obtained without multiplying through by the window, and is the combined "limit" profile expected for no window-function contamination. Both have been normalized to the same maximum power to emphasize visually the introduction of additional background.

\section{Impact on background-to-signal ratio $\beta$}

\subsection{Overview of impact of multi-station window}

The introduction of a temporal window function generated by a multi-station network results in power density in the frequency domain being redistributed from the principal resonant peaks into: sidebands, which are the result of quasi-diurnal gap structure in the window; and a complicated background that arises from quasi-random gap structure due to inclement weather and occasional instrumental breakdown. Both are illustrated in Fig. 2.

Here, we show part of an artificial $p$-mode spectrum that contains an $\ell=2 / 0$ pair. The plotted spectrum is in fact the result of the incoherent addition (in the frequency domain) of one thousand independent realizations of the dataset. The dashed line illustrates the mean spectrum obtained by co-adding data with a 100-per-cent fill: this tends to the combined limit profile expected for no window-function contamination. The effects of modulating each set by a BiSON window with a duty cycle of $D=0.75$ are shown as the solid line. The introduction of the window function results in power being re-distributed from the mode (signal) peaks into the background. As a result the power density in the signal is reduced, while that in the background increases. (Note that in Fig. 2 both spectra are shown normalized to the same maximum power; this has been done to emphasize visually the introduction of additional background.) The re-distribution of power density between the modes, and the resulting complicated background this produces, is most evident. The impact this has on the background-to-signal ratio 
is illustrated in the various panels of Fig. 3. First, we consider the reference case of a 100-per-cent fill (upper two panels).

The upper left-hand panel shows the radial-mode $(\ell=0)$ heights (points with errors) and background (dotted line) extracted from fits to a $10-\mathrm{yr}$ artificial spectrum with $D=1.00$. A set of this length gives an intrinsic resolution of $\sim 3 \mathrm{nHz}$ in the frequency domain. The solid and dashed lines follow the input height and input "pink" background respectively. At lower frequencies - where the mode peaks are narrow - the fitted background follows the input trend. This implies that in the real data the solar noise (which arises from different scales of granulation), and any instrumental contribution, should dominate at these frequencies. Over the main part of the $p$-mode spectrum these terms decrease in magnitude. Here, there is an additional contribution to the fitted background that comes from the overlapping tails of the Lorentzian-like peaks (which are more heavily damped in this part of the spectrum). This is apparent in the artificial data at frequencies above $\sim 2500 \mu \mathrm{Hz}$.

The triangles in the upper right-hand panel follow the resulting fitted background-to-signal ratio $\beta_{n \ell}$. This we define as the ratio of the background fitted around a given mode, $B$, to its fitted height, $H_{n \ell}$, i.e.,

$\beta_{n \ell}=B / H_{n \ell}$.

The dashed line is the input $\beta_{n \ell}$ given by the ratio of the pink background and actual mode height.

The middle panels of Fig. 3 show the same parameters but now for the case of a 10-yr artificial dataset modulated by the six-station BiSON window. This has an associated duty cycle of $D=0.71$. The middle left-hand panel has been scaled to conserve Parseval's Theorem: as such it shows the raw heights fitted in a spectrum scaled in this manner. That they underestimate the input height is only to be expected since power density has been removed (aliased) from the principal peaks by the window function. A fitted estimate of the input height can nevertheless be recovered by dividing through by the fill (to which the fraction of power redistributed is proportional). These estimates are plotted as diamonds (we return to a discussion of any bias in these in Sect. 4 below).

Were diurnal sidebands to be the only structures into which power is aliased - these being located at a reasonable separation from the main peaks - the implied increase in the backgroundto-signal ratio, $\beta$, would be only of order $\sim 30$ per cent. However, as indicated already this is unfortunately not the case. Power is redistributed also into a complicated structure in the near vicinity of the modes giving a substantial increase in the background level. As a result, $\beta$ is seen to increase - here, by up to an order of magnitude - over a large part of the spectrum (middle right-hand panel of Fig. 3). The effect is most prevalent for strong and wide (heavily damped) mode peaks.

We also show in the lower two panels the fitted $\beta_{n \ell}$ (triangles) extracted from fits to the real 10-yr BiSON (lower left-hand) and 800-d GOLF (lower right-hand panel) sets. The dashed line in each of these (included for reference) is the input $\beta_{n \ell}$ given by the ratio of the pink background and input mode height of the artificial data. The increased background-to-signal ratio in the BiSON data is seen to match reasonably well that of the artificial $D=0.71$ spectrum in the middle right-hand panel.
It is apparent however that at lowest frequencies the $\operatorname{BiSON} \beta$ is higher than in the artificial data. We intend to address the question of mode visibility and detectability at low frequencies in a forthcoming paper (Chaplin et al., in preparation).

The bottom right-hand panel reveals that the GOLF $\beta$ follows closely that of the artificial $D=1.00$ set. Although modes are prominent in these data outside the frequency range plotted, we have restricted ourselves to those fits for which a reliable estimate of the "shape" or form of the mode profile could be extracted (Thiery et al. 2000). The excellent agreement of the GOLF and artificial $\beta$ here was not achieved directly by design: the simulation parameters are the result of modelling the variation of the resonant and background characteristics in the BiSON, not the 100-per-cent-fill GOLF, data.

We should add at this point that the difference in the length of the GOLF and BiSON data is not a contributory factor to the difference in the background-to-signal level. The observed $\beta_{n \ell}$ should be independent of the observing time, $T$, provided the uncovered modes are prominent ${ }^{1}$ and well resolved, and the noise characteristics of the data remain unaltered (as demonstrated in Appendix A). This is emphatically not, however, an argument against the need for longer datasets. This is because the precision with which the parameters can be determined increases as more data are collected, i.e., as $T^{1 / 2}$ for that in the frequencies, widths and heights of resolved modes (see Chaplin et al. 2002). Furthermore, there is a clear need for extended continuity in order to uncover the weak, unresolved (narrow) modes expected at low frequencies (Chaplin et al., in preparation).

\subsection{Formalism for variation of $\beta$ with fractional fill}

Some examples of the impact on the GOLF data of the introduction of the four hundred BiSON window functions (cf. Sect. 2.1) are shown in Fig. 4. Here, we plot fitted $\beta_{n \ell}$ as a function of the fractional fill of each window function, $D$, for three radial modes ( $n=16,20$ and 24).

Chaplin et al. (2002) attempted to model quantitatively the variation of background-to-signal ratio with fractional fill, $D$. They did so by expressing the $\beta_{n \ell}$ observed in a given mode $(n, \ell)$ as:

$\beta_{n \ell}^{D}=\frac{\Delta v_{n \ell} \cdot e_{n \ell} \cdot(1-D)+\beta_{n \ell}^{1}}{D}$

where in the above $\beta_{n \ell}^{1}$ is the background-to-signal ratio at $D=1.00$. This was formulated under the assumption that both the term in the denominator - which reflects the reduction in signal height that results from aliasing of power into the diurnal sidebands - and that in the numerator - which reflects the introduction of the broad-band background, with the coefficient $e_{n \ell}$ fixing the size of this - depend linearly on $D$. The expression is pertinent to any dataset length provided the mode it describes is well resolved (Appendix A). Furthermore, the

\footnotetext{
1 At high background-to-signal ratios, when a mode may be barely visible, the variance of (or noise on) the background becomes an important factor in determining detectability.
} 

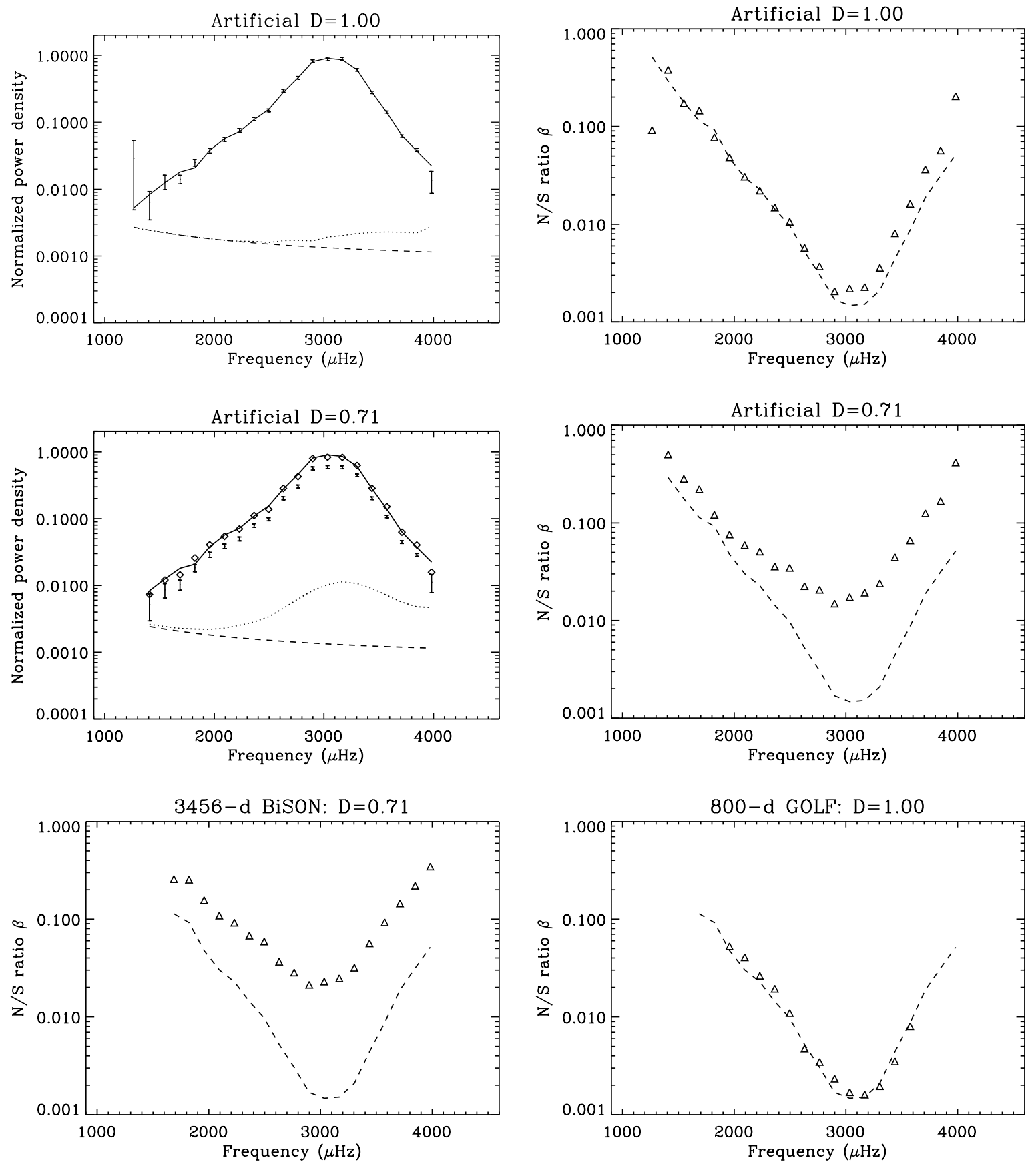

Fig. 3. The impact on both artificial and real low- $\ell$ data of the introduction of a window. The upper left-hand panel illustrates results from fitting a 10 -yr artificial spectrum with no window function (i.e., $D=1.00$ ). Shown are: the fitted $\ell=0$ height (points with errors) and background (dotted line); and the actual (input) height (solid line) and "pink" background (dashed line). The middle left-hand panel shows the same parameters but now for the case of an artificial dataset modulated by the $10-\mathrm{yr}$, six-station BiSON window (with $D=0.71$ ). An estimate of the input height that allows for the impact of the window can be recovered by dividing each raw, fitted height by $D$. These estimates are plotted as diamonds. The other panels show the fitted background-to-signal ratio, $\beta_{n \ell}$, (triangles) extracted from fits to both artificial datasets and also the real 10-yr BiSON (lower left-hand) and 800-d GOLF (lower right-hand panel) sets. The dashed line in each of these (included for reference) is the input $\beta_{n \ell}$ given by the ratio of the pink background and input mode height of the artificial data (which match well the fitted $\beta_{n \ell}$ of GOLF).

$e_{n \ell}$ are characteristic of a given instrumental dataset, and once determined are fixed for that instrument regardless of $D$.

Here, we have used the window-function-modulated GOLF sets to estimate $e_{n \ell}$ for radial modes in real data. Best fits of the model (Eq. (6)) are rendered as a solid line for each of the $n$ shown in the two upper, and lower left-hand, panels of Fig. 4.
The extracted best-fit coefficients are plotted as a function of frequency in the lower right-hand panel. The high, out-of-line datum is the result of the deleterious influence of the first diurnal sideband of the nearest sectoral component of the neighbouring $\ell=2$ mode. 

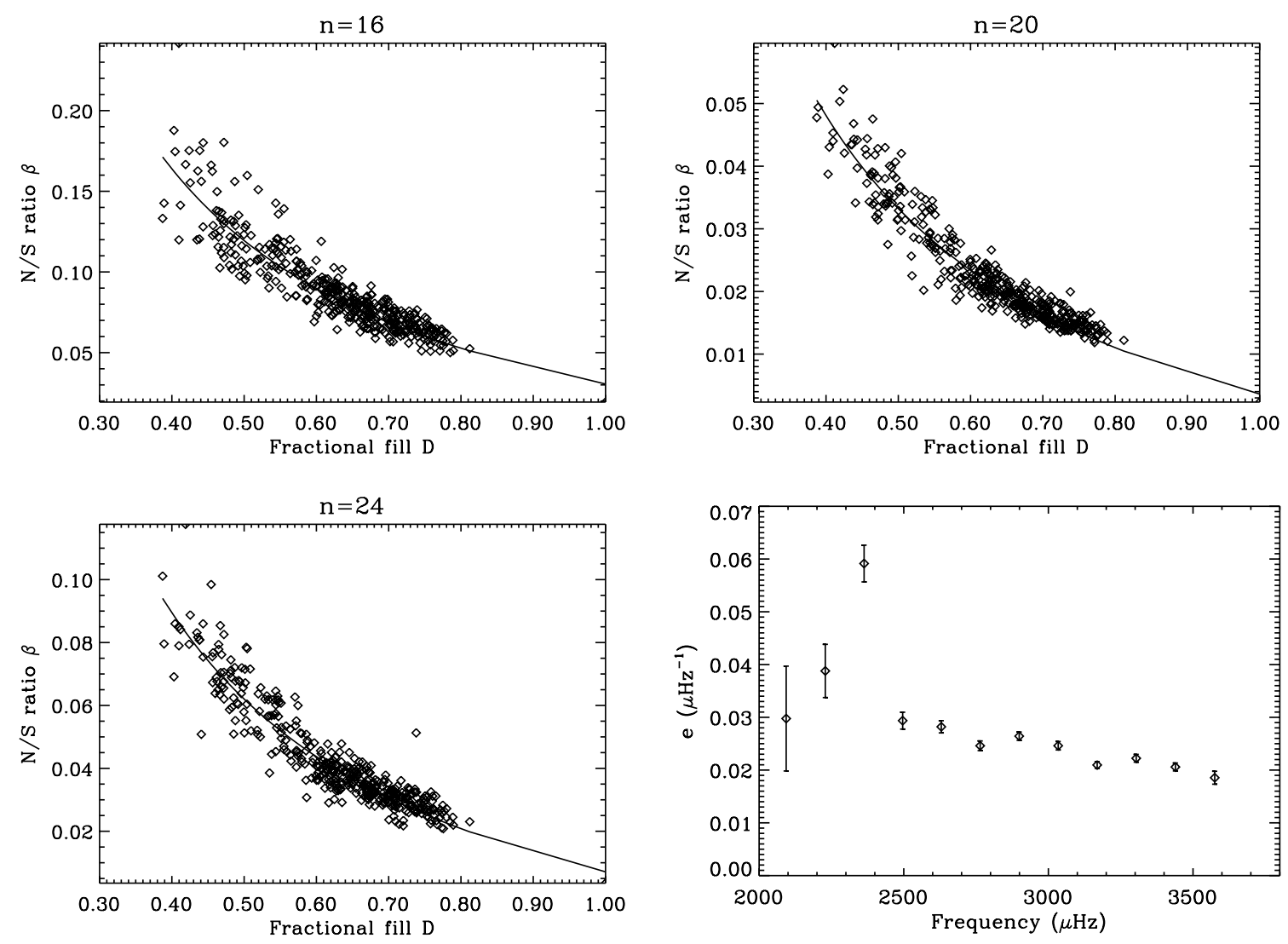

Fig. 4. Examples of the impact on the GOLF data of the introduction of the four hundred BiSON window functions (cf. Sect. 2.1). Plotted,as a function of fractional fill $D$, are the fitted background-to-signal ratio, $\beta_{n \ell}$, for three radial modes $(n=16,20$ and 24 in upper left and right, and lower left-hand panels). Best fits of the Chaplin et al. (2002) model, which describes the change in $\beta$ with fill, are rendered in each panel as a solid line. Lower right-hand panel: best-fit estimates (as a function of frequency) of the free parameter in the model, $e_{n \ell}$.

The coefficients in the figure allow an easy and reliable calculation to be made of the $S / N$ expected at any fill. Similar values are returned for the artificial data.

\section{Impact on bias and uncertainty of $\Delta v, H$ and $P_{Q}$}

\subsection{Bias}

While the GOLF series does not let us test directly the accuracy of the fitted parameters with respect to the underlying, solar value - this of course presumes a priori knowledge we do not have - it does nevertheless allow us to assess quantitatively changes to the fitted $D=1.00$ parameters under conditions of varying fill. That is: we can assess the bias introduced by various window functions where this is determined with respect to the $D=1.00 \mathrm{GOLF}$ value. In the second part of the section we make use of our artificial spectra in an effort to address the likely precision of the complete GOLF set.

The two panels of Fig. 5 show the fitted width and height returned at $\ell=0, n=17$ when the various $800-\mathrm{d}$ BiSON window functions are used to modify the GOLF time series. The parameters are shown normalized to the fitted value given for the continuous, 100-per-cent fill set. The presence of bias in the fitted estimates is most evident: here, both parameters increasingly overestimate the $D=1.00$ value as the fill decreases.
Similar alarming trends are present also at the other $n$. The effect is quantified in Fig. 6 .

Here, we plot the implied fractional change in both mode parameters for every 10-per-cent drop in fill (i.e., $\delta D=-0.1$ ), under the assumption of a linear dependence over the range of $D$ considered. Shown also is the variation implied in the acoustic noise generation rate, $P_{\mathrm{Q}}$, assuming it to have been calculated from the width and height parameters (this being proportional to the product of the square of the width, $\Delta v_{n \ell}$, and the height, $H_{n \ell}$ ).

The implied bias in the width and noise generation rate is most severe at the centre of the $p$-mode range (i.e., at $\approx 3000 \mu \mathrm{Hz}$ ) where both trend-lines are peaked; while that in the height shows a minimum at this location. This is where the modes appear strongest and the window function contamination is most prevalent. Unfortunately, this coincides also with a particularly interesting part of the spectrum where the observed linewidths not only plateau but also decrease in magnitude. The resulting dip is believed to be caused by non-adiabatic, radiative processes in the superadiabatic layer of the convection zone (e.g., see Houdek et al. 1998, for a general overview of mode damping). Houdek et al. (2001) showed that its characteristics could be altered noticeably by changing the preferred horizontal length scale of the convective flow; in so doing they attempted to model variations that are seen in the linewidths over the activity cycle (e.g., Chaplin et al. 2000; Komm et al. 2000). 

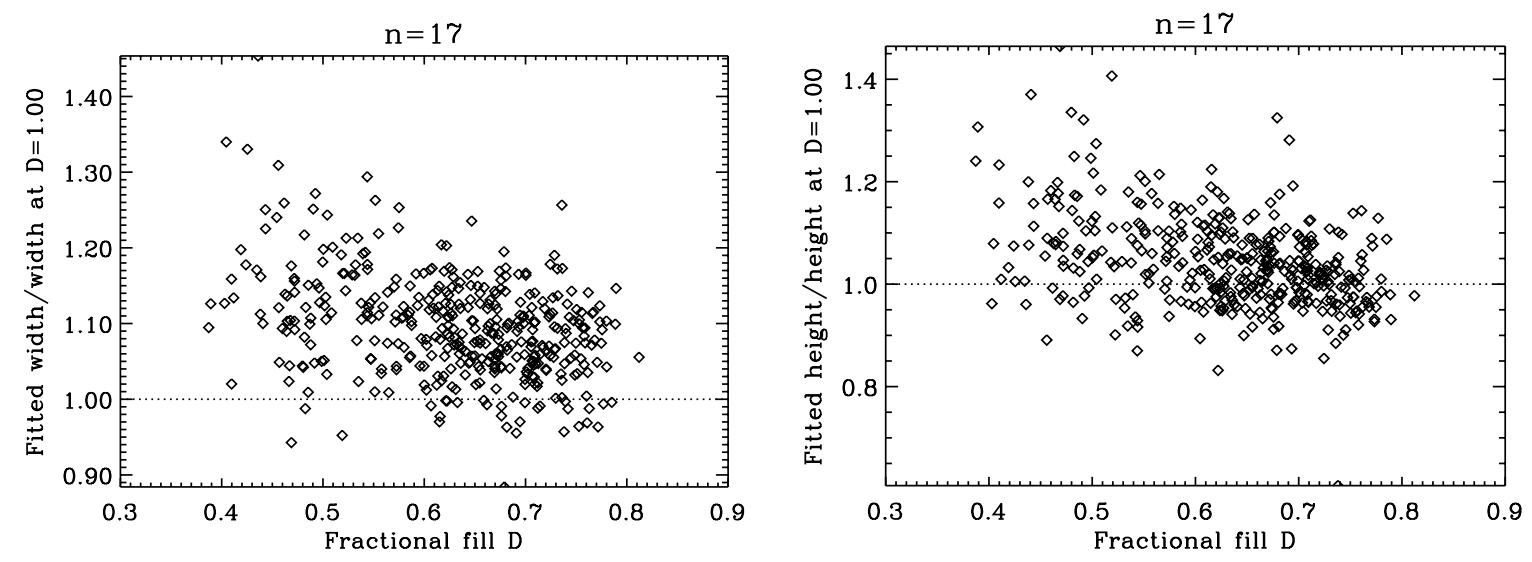

Fig. 5. Fitted width and height at $\ell=0, n=17$ returned when the GOLF data are modulated by BiSON window functions of differing fills, $D$. The parameters are shown normalized to the fitted value in the complete, $D=1.00$ set.
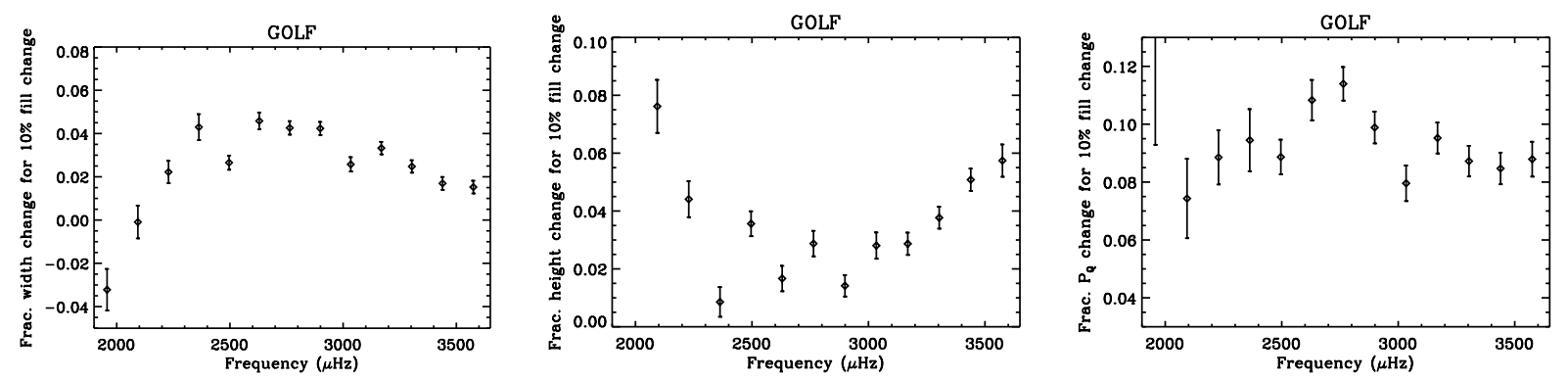

Fig. 6. The fractional change in the fitted width, height and acoustic noise generation rate (proportional to product of width squared times height) of radial modes in the 800-d GOLF set for each 10-per-cent decrease in duty cycle (i.e., $\delta D=-0.1$ ).

These are observed to be peaked also at just under $\sim 3000 \mu \mathrm{Hz}$. Clearly, the window-induced bias complicates not only the task of measuring accurately the characteristics of the dip, but also any variation in its location and depth from data spread across the activity cycle that may have differing fill.

The bias estimates in Fig. 6 measure the gradient, or sensitivity, of the fitted parameters to $D$. However, they are not a direct estimate of the amount by which fits overestimate the underlying solar value. We can place limits on this, provided we believe the fitted uncertainties on the $D=1.00$ GOLF parameters reflect the precision of each measure. To help judge the likelihood of this we make use of artificial data for which we have a priori knowledge of the actual parameters.

In the various panels of Fig. 7 we show the fractional difference between the fitted width, height and acoustic noise generation rate parameters and their respective input values for analyses of: (i) a 10-yr artificial dataset with $D=1.00$ (top row); (ii) the same 10 -yr set, now modulated by the $D=0.71 \mathrm{BiSON}$ window (middle row); and (iii) an 800-d portion of the set chosen to coincide with an $800-\mathrm{d}$ window having $D=0.71$ also (bottom row). The plotted triangles with associated uncertainties show those differences extracted at $\ell=0$, while the dotted and dashed lines are the corresponding trend lines at $\ell=1$ and 2 .

The fitted values from the continuous set show no significant evidence of bias over most of the spectrum. However, there is evidence of increased scatter - giving poorer estimates - at low frequencies where the modes are somewhat narrower and the background-to-signal ratio higher. The results with the window imposed show significant variation with characteristics very similar to those of the GOLF trends plotted in Fig. 6. The bias would also appear to be more severe in the shorter, lower-resolution set.

If - given the $D=1.00$ results here in Fig. 7 - we assume tentatively that the GOLF parameter estimates from its complete set are unbiased also, the GOLF sensitivity gradients in Fig. 6 can then be used to estimate the bias at $D=0.71$. This is shown in Fig. 8.

The solid line in each panel is the bias inferred by multiplying the gradients in Fig. 6, which were normalized to $\delta D=-0.1$, by a factor 2.9 (i.e., to give $D=1.0-2.9 \times$ $0.10=0.71$ ). The dotted lines, which mark the $1 \sigma$ uncertainty in the absolute location of this, are the fitted error bars on the $D=1.00$ GOLF parameters. They reflect the assumed uncertainty in the precision of the GOLF values. Plotted also are the 800-d artificial results from the bottom row of Fig. 7.

The level of agreement between the GOLF-inferred and artificially-inferred bias is quite striking. While the artificial width-bias appears to bracket nicely (within errors) its GOLF counterpart, the artificial height-bias is more negative (perhaps by as much as $1 \sigma)$. This inevitably impacts also on the $P_{\mathrm{Q}}$ estimates. It is most likely that a slight mismatch in the noisebackground characteristics of the artificial and GOLF data is enough to account for the difference. Nevertheless, the results here are encouraging for several reasons. 

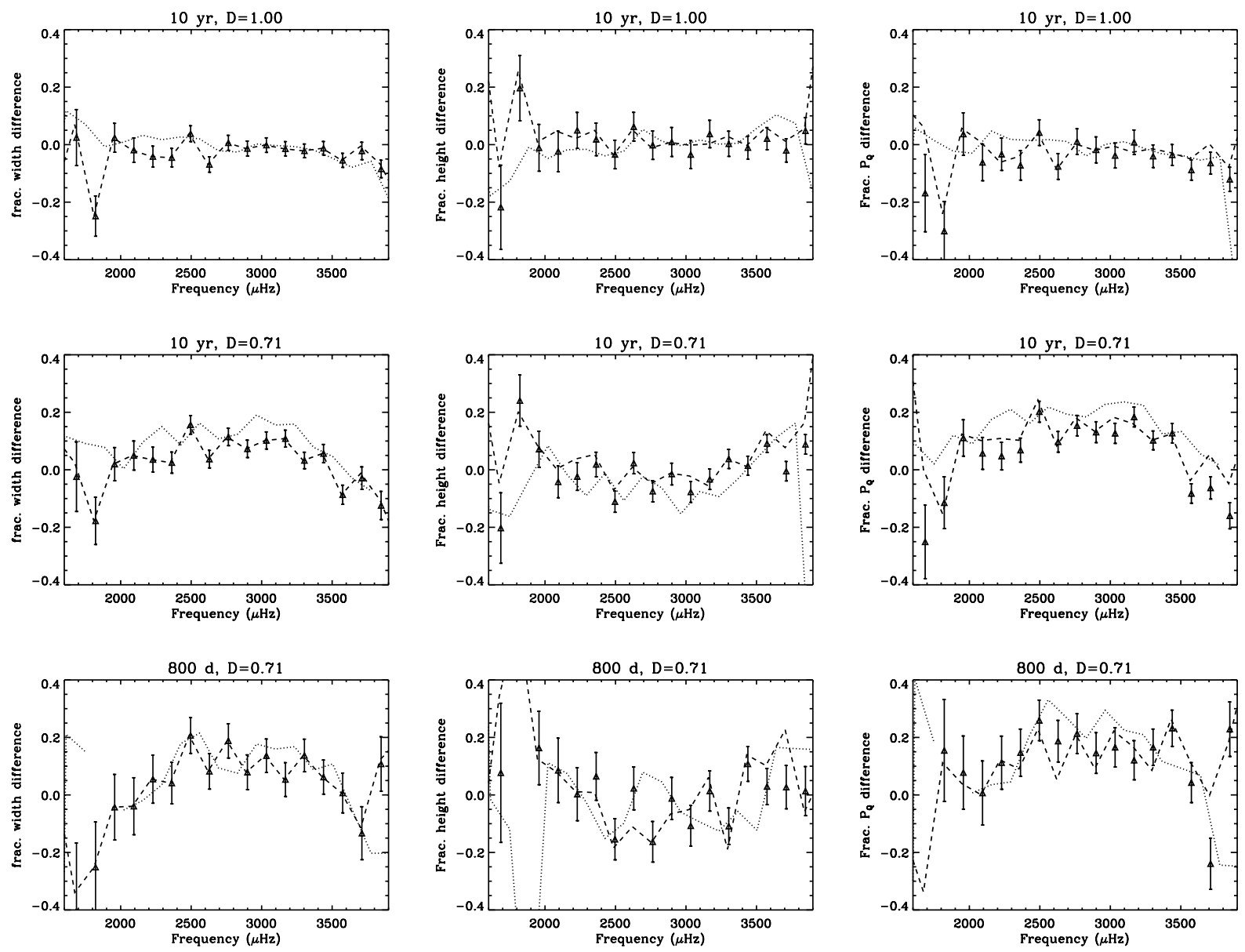

Fig. 7. Fractional difference between the fitted width, height and acoustic noise generation rate $\left(P_{\mathrm{Q}}\right)$ and the actual, input values for radial modes in artificial datasets (data with errors). The dotted and dashed lines show the corresponding trend lines at $\ell=1$ and 2 respectively. Top row: results from a 10-yr dataset with $D=1.00$. Middle row: results from the same 10-yr set, now modulated by the $D=0.71 \mathrm{BiSON}$ window. Bottom row: those from an 800-d portion of the set chosen to coincide with a window of the same length having $D=0.71$.

First, they imply that the $D=1.00$ GOLF error estimates may be a reasonable guide to the precision of the parameters. Second, they indicate that the artificial data are more-thanlikely a robust tool for testing the analysis of helioseismic data; and third, they suggest therefore that any empirical window corrections derived from comprehensive artificial simulations are probably sound. However, there remains of course the possibility that unforeseen, or poorly modelled, features of the real data may compromise to some extent the veracity of the simulations.

\subsection{Uncertainty}

The variation of the $\ell=0, n=17$ fractional width and height uncertainties, $\operatorname{dlog}_{\mathrm{e}}\left(\Delta v_{n \ell}\right)$ and $\operatorname{dlog}_{\mathrm{e}}\left(H_{n \ell}\right)$ respectively, returned from fits to the GOLF data are plotted in Fig. 9. These are the formal errors derived from the Hessian matrix of each fit. They are seen to increase in magnitude by about $\approx 30$ per cent over the range of tested $D$. Plotted also in the figure (dashed line) are estimates of each uncertainty based on the expressions of Toutain \& Appourchaux (1994).

In addition to complete formalisms for each expected error Toutain \& Appourchaux derived also simpler, approximate (and therefore more usable) versions that we have modified here with a correction factor $c_{n \ell}^{\text {obs }}$. These "semi-empirical" expressions are:

$d \log _{\mathrm{e}}\left(\Delta v_{n \ell}\right)=c_{n \ell}^{\mathrm{obs}} \times\left(\frac{\left[\left(1+\beta_{n \ell}\right)^{1 / 2}+\beta_{n \ell}^{1 / 2}\right]^{4}}{\pi \Delta v_{n \ell} T}\right)^{1 / 2}$

and

$d \log _{\mathrm{e}}\left(H_{n \ell}\right)=2 c_{n \ell}^{\mathrm{obs}}\left(\frac{\left(1+\beta_{n \ell}\right)^{3 / 2}\left[\left(1+\beta_{n \ell}\right)^{1 / 2}+\beta_{n \ell}^{1 / 2}\right]}{\pi \Delta v_{n \ell} T}\right)^{1 / 2}$.

We found that (as indicated in Fig. 9) the trend in the fitted uncertainties matched quite well the predicted form of the semiempirical equations. However, at each $n$ a correction factor of typical size $c_{n \ell}^{\text {obs }} \approx 0.85$ was required in order to give a better match in absolute magnitude. It is worth adding that the precise magnitude of the returned uncertainties is affected by the number of mode parameters one seeks to estimate during fitting. Nevertheless, Eqs. (7) and (8) would appear to provide a useful guide to the form of the variation of the uncertainties with $D$, and reasonable estimates of the actual parameter uncertainties themselves. 

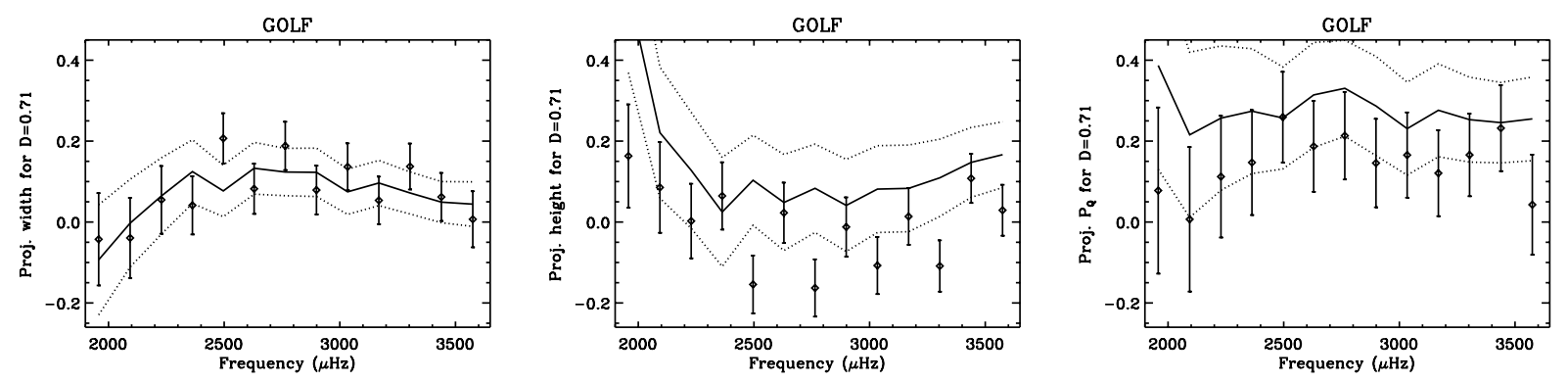

Fig. 8. An estimate of the absolute bias (i.e., with respect to the actual, solar value) of the fitted width, height and acoustic noise generation rate, for an 800-d set with $D=0.71$. The solid line in each panel is the bias estimated from analyses of the GOLF data, with the dotted lines the $1 \sigma$ uncertainty envelope on the absolute location of this. Plotted also (data with errors) are the artificial, 800-d estimates from the bottom row of Fig. 7.
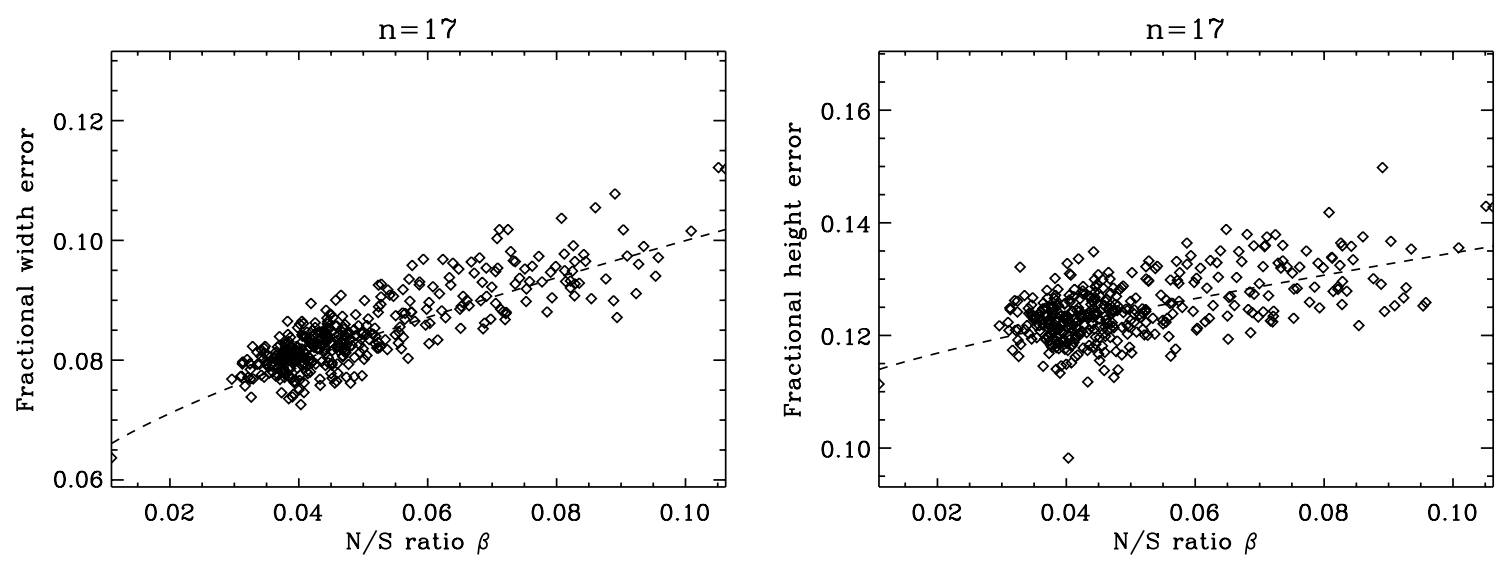

Fig. 9. Formal fractional uncertainties in the fitted width and height at $\ell=0, n=17$ when the GOLF data are modulated by BiSON window functions of differing fills, $D$. The dashed line is a scaled prediction based on the semi-empirical-Hessian formula of Toutain $\&$ Appourchaux (1994).

The implied reduction in precision with decreasing $D$ is sufficient to impact negatively on, for example, studies that attempt to uncover $(\ell, m)$-dependent variations in width and height over the solar cycle. Initial indications are that the size of the differences in these at low $\ell$ are comparable to the fractional uncertainties in the parameters themselves (Toutain \& Kosovichev 2002; Chaplin et al., in preparation).

Acknowledgements. We are extremely grateful to the GOLF team for allowing us to use their data. With regard to BiSON, we are, as ever, indebted to H. K. Williams, J. Allison and R. Bryan for their technical and analysis support in Birmingham and to former colleagues, in particular C. P. McLeod and J. Litherland. We would also like to thank P. Fourie and the late R. Stobie at SAAO; the Carnegie Institution of Washington; the Australia Telescope National Facility (CSIRO); E. J. Rhodes (Mt. Wilson, California); and members (past and present) of the IAC, Tenerife. SOHO is a mission of international cooperation between ESA and NASA. BiSON is funded by the UK Particle Physics and Astronomy Research Council. ST acknowledges the support of a PPARC research fellowship.

\section{Appendix A: Variation of background-to-signal ratio $\beta$ with time}

Consider a time series of length $T$, sampled at a cadence of $\Delta t$. We consider the example of a simple set that contains two components: first, a stochastically excited $p$ mode with meansquare power $V_{n \ell}^{2}$ that is damped with constant $\eta_{n \ell}$; and second, a normally distributed ("white") noise source with zero mean and sample standard deviation $\sigma$. When the power spectrum of the time string is computed this yields a peak of height $H_{n \ell}$ against a background level $B$. The mean level $B$ which satisfies Parseval's theorem in the frequency domain is:

$B=2 \sigma^{2} \Delta t$,

where we take a power spectral density (power per Hertz) scaling.

The way in which $H_{n \ell}$ relates to the other modal parameters depends upon whether the mode is "over"- or "under"sampled. In the oversampled regime, the coherence, or lifetime of the mode, $\tau_{n \ell}$, is smaller than the observing time $T$. The efolding lifetime is related to the damping constant $\eta_{n \ell}$ and the frequency-domain linewidth, $\Delta v_{n \ell}$ by:

$\tau_{n \ell}=1 / \eta_{n \ell}=1 / \pi \Delta v_{n \ell}$.

When $T \gg \tau_{n \ell}$, the time series will then extend over a whole series of independent realizations and the modal peak will be well resolved (i.e., lie across many bins) in the frequency domain. The analysis assumes explicitly that the underlying mode profile can be described as a Lorentzian function. Even though the modal peaks are asymmetric at low $\ell$ (Toutain et al. 1998; 
Chaplin et al. 1999; Thiery et al. 2000), the magnitude of this is so small as to make a Lorentzian description sufficiently accurate for our purposes here. The maximum height (power density) of the underlying limit form of the mode in the power spectrum (taking a mean square scaling) is then:

$H_{n \ell}=\frac{2 V_{n \ell}^{2}}{\pi \Delta v_{n \ell}}$.

Were $T$ to be reduced to such an extent that $T \ll \tau_{n \ell}$, the underlying profile would be so narrow as to confine all power within one bin. Here, we assume that the signal is commensurate with the window function. In the resulting undersampled regime we have conditions that therefore tend toward those expected of a simple undamped sine wave ${ }^{2}$, where $H_{n \ell} \sim V_{n \ell}^{2} T$. With reference to Eq. (A.3), the changeover to this regime occurs when:

$T=2 \tau_{n \ell}=2 / \pi \Delta v_{n \ell}$.

So, a full description of $H_{n \ell}$ in terms of the other parameters is:

$H_{n \ell}= \begin{cases}\left(2 V_{n \ell}^{2}\right) /\left(\pi \Delta v_{n \ell}\right) & \text { for } T \gg 2 \tau_{n \ell} \\ \sim V_{n \ell}^{2} T & \text { for } T \ll 2 \tau_{n \ell} .\end{cases}$

The background-to-signal ratio, $\beta_{n \ell}$ at which the mode appears is therefore:

$\beta_{n \ell}=B / H_{n \ell}= \begin{cases}\left(\pi \sigma^{2} \Delta v_{n \ell} \Delta t\right) /\left(V_{n \ell}^{2}\right) & \text { for } T \gg 2 \tau_{n \ell} \\ \sim\left(2 \sigma^{2} \Delta t\right) /\left(V_{n \ell}^{2} T\right) & \text { for } T \ll 2 \tau_{n \ell} .\end{cases}$

Equation (A.6) shows that while the mode is undersampled (i.e., in the regime for which $T \ll 2 \tau_{n \ell}$ ) the backgroundto-signal ratio $\beta_{n \ell}$ decreases as $T$ increases. However, given fixed noise and mode characteristics, and a chosen observational cadence, $\beta_{n \ell}$ is constant and independent of $T$ once the mode is oversampled and resolved (i.e., for $T \gg 2 \tau_{n \ell}$ ). As demonstrated by Chaplin et al. (2002), this is the case for the vast majority of those low- $\ell$ $p$ modes uncovered and studied to date (save for several uncertain detections at frequencies below $1000 \mu \mathrm{Hz}$ ).

\section{References}

Bertello, L., Henney, C. J., Ulrich, R. K., et al. 2000, ApJ, 535, 1066 Chaplin, W. J., Elsworth, Y., Howe, R., et al. 1996, Sol. Phys., 168, 1 Chaplin, W. J., Elsworth, Y., Howe, R., et al. 1997, MNRAS, 287, 51

Chaplin, W. J., Elsworth, Y., Isaak, G. R., Miller, B. A., \& New, R. 1999, MNRAS, 308, 424

Chaplin, W. J., Elsworth, Y., Isaak, G. R., Miller, B. A., \& New, R. 2000, MNRAS, 313, 32

Chaplin, W. J., Elsworth, Y., Isaak, G. R., et al. 2001, MNRAS, 327, 1127

Chaplin, W. J., Elsworth, Y., Isaak, G. R., Miller, B. A. \& New, R. 2002, MNRAS, 330, 731

Christensen-Dalsgaard, J. 1989, MNRAS, 239, 977

Gabriel, A. H., Charra, J., Grec, G., et al. 1997, Sol. Phys., 175, 207

Houdek, G., Chaplin, W. J., \& Elsworth, Y. 1998, A\&G, 39, 30

Houdek, G., Balmforth, N. J., Christensen-Dalsgaard, J., \& Gough, D. O. 1999, A\&A, 351, 582

Houdek, G., Chaplin, W. J., Appourchaux, T., et al. 2001, MNRAS, 327,483

Houdek, G. 2002, in Radial and non-radial pulsations as probes of stellar physics, Proc. IAU Symp 185, ed. C. Aerts, \& T. Bedding, PASP, 447

Jiménez, A., Roca-Cortés, T., Severino, G., \& Marmolino, C. 1999, ApJ, 525, 1042

Komm, R., Howe, R., \& Hill, F. 2000, ApJ, 531, 1094

Libbrecht, K. G. 1992, ApJ, 387, 712

Nigam, R., \& Kosovichev, A. G. 1998, ApJ, 505, L51

Pallé, P., Régulo, C., Roca Cortés, T., et al. 1999, A\&A, 341, 625

Samadi, R., Goupil, M.-J., \& Lebreton, Y. 2001, A\&A, 370, 147

Stein, R. F., \& Nordlund, A. 2001, ApJ, 546, 585

Thiery, S., Boumier, P., Gabriel, A. H., et al. 2000, A\&A, 355, 743

Toutain, T., \& Appourchaux, T. 1994, A\&A, 289, 649

Toutain, T., Appourchaux, T., Fröhlich, C., et al. 1998, ApJ, 506, L147

Toutain, T., \& Kosovichev, A. G. 2002, in SOHO 11 Symp. From Solar Min to Max: Half a Solar Cycle with SOHO, ed. C. Fröhlich, \& A. Wilson, ESA SP-508, 103

\footnotetext{
${ }^{2}$ Here, we refrain from using an exact equality in the equation since the actual form the peak will take - the sinc-squared function of the envelope of the time series - will lead to some, albeit minimal, power leakage.
} 